\title{
Analysis and Design of Deep Learning Algorithms for Retinal Image Classification for Early Detection of Diabetic Retinopathy
}

\author{
Amita Meshram \\ Nagpur
Dr. Deepak Dembla
Jaipur \\ Dr. Reema Ajmera \\ Jaipur
}

\begin{abstract}
This paper presents a far reaching survey of the standard and use of deep learning in retinal image investigation. Many eye ailments regularly lead to visual impairment without legitimate clinical determination and clinical treatment. For instance, diabetic retinopathy (DR) is one such illness in which the retinal veins of natural eyes are harmed. The ophthalmologists analyze DR dependent on their expert information that is work escalated. With the advances in image preparing and man-made reasoning, Personal Computer vision-based methods have been applied quickly and broadly in the field of clinical images investigation. The important deep learning algorithms such as CNN Convolution Neural Network, ConvNet based algorithm, LCD net and Deep CNN, their working and main features of some of these standard Deep Learning algorithm are analyzed in detailed. Proposed algorithm will become more reliable accurate by introducing new features as well as better quality input by using advance algorithm of image processing.
\end{abstract}

Keywords: Diabetic Retinopathy, Convolution neural network, Recurrent neural network, Reinforcement Learning to Neural Networks.

\section{Introduction}

Eye Blindness is a difficult issue happening all inclusive. The significant reasons for visual impairment are Diabetic Retinopathy (DR),Glaucoma etc. In Ophthalmology, deep learning has been applied to fundus retinal pictures accomplishing vigorous grouping execution in the recognition of eye illnesses like Diabetic Retinopathy, the Glaucoma-like plate, age-related Macular Degeneration. Man-made brainpower based profound learning calculation is fitting for the identification of eye illnesses at a beginning phase.

In Beginning phases of these illnesses are hard to distinguish, and whenever left untreated, this can make permanent harms vision. Diabetic Retinopathy is caused for the most part because of the persistent diabetic stage in an individual and is seen as the significant reason for visual deficiency. Diabetic Retinopathy is because of harms in the retinal veins. At the point when sugar level extended in blood that may cause blocks in veins that help the retina and stop its blood deftly Because of that, the eye produces fresh blood vessels. These as of late creating veins don't grow appropriately so it spill fastly and easily.K V Maya; K S Adarsh (2019).

Manual exertion gets diminished due to automatic program that is incredibly required and cost in this strategy is generally more. Recognition in retinal pictures ought to be modified by utilizing advanced picture catching and picture preparing procedures to make it cost-effective. In Diabetic Retinopathy veins that help in gainful the retina start radiates liquid and blood on the retina which gives in visual highlights known as sores like cotton fleece spots, microaneurysms, hard exudates, vein areahemorrhages. These injuries with the life systems of the retina can be appeared in fig 1. A.Guptaa , R.Chhikarab (2018). These all highlights will be separated by the image extraction strategy and by applying the proposed profound learning calculation that will bring about better class wise precision expectation.

In present day times, for clinical image testing and finding of different ailments, there has been large enthusiasm for utilizing profound learning. For instance, modernized examination of color retinal pictures for diabetic retinopathy finding has been considered; have as of late accomplished broadly better results in computer vision and clinical picture investigation for picture arrangement and item identification A.Shah, S.Lynch et.al.(2018) 


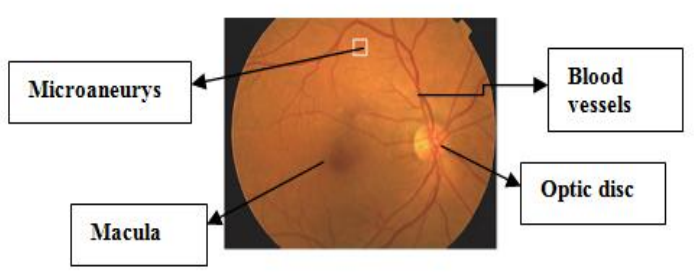

Figure1. Various lesions of retina

In image characterization the classification between images is an extremely simple assignment for individual, yet it is troublesome and significant task for computer. images are marked into predefine classes by Image arrangement.

Ordering a handwritten digit and allocating a name to a picture of a face are the example of (multiclass classification) and marking an x-ray as cancer or not is the example of (binary classification).Different structures of image classifications are i) Image Preprocessing - It is refinement of image data, and an improvement of image data by suppressing unwanted distortions. It includes some stages for image preprocessing are read image, resize image,data augmentation which includes Gaussian Blurring, Rotation, Translation Histogram Equalization, etc. ii) Recognition of an object - It refers localization and segmentation of the image, and detect the location of the image. iii) Feature extraction- Images are divided into different features, identify the pattern of images according to features from dataset and divided into different classes and train the model according to that. iv) Classification of the object - By using classification method noticed objects are categorize into predefine classes and matches the image designs with the goal pattern.

Various image classification techniques are Decision Trees, Support vector Machine. SVM is a supervised machine learning algorithm they are used for both regression and classification problems. Decision trees are rule based methods to shows result in the form of true/false or yes/ no of class labels at each level. It involves of 3 parts: separating the nodes, finding the terminal nodes. The k-nearest neighbor classification algorithm is used for finding common class which depends on categorizes unknown data points and the distance among feature vectors. The classification is very important for detection of diabetic Retinopathy.

On PASCAL VOC 2007 data-set author applied convolutional neural system (CNN) model pre-trained on image net is utilized for classification of images. Classification works genuinely fine with the littlest measure of calculation time and less machine assets and to recover performance of deep learning CNN model, the transfer learning approach is utilized. The execution has been estimated by Deep learning CNN model. By using Supervector coding of local image descriptors method, SVM technique, and Locale Ranking SVM strategy, are used for test results and compared with obtained result, which tried with the PASCAL VOC 2007 data set. For an Image classification the last results assess the Deep Learning algorithm as a bestW AEzat, M MDessoukyand N A Ismail (2019)

There are some challenges and issues in previous work that has been done so far. Few features are not considered yet. Our study focuses on such features of retinal image that are not considered by earlier researchers. In preveious work there is no such algorithm which focuses on overall accuracy of each class.

\section{Review Of Literature}

Authors T. Li a,b et al., (2019) assessed best in class deep learning algorithm on accumulated images, including images classification, segmentation and detection. Lesion segmentation and recognition are exceptionally hard to identify. These algorithms achieve deficiently on lesion segmentation and recognition. They apply deep learning model on dataset named DDR (Diabetic Retinopathy Dataset) to examining the clinical applications. Authors C. Lam, MD, D.Yi , M.Guo, T.Lindsey (2018) demonstrated the utilization of convolutional neural systems (CNNs) on shading fundus images for the acknowledgment undertaking of diabetic retinopathy. They also investigated multinomial characterization models, and show that errors principally happen in the misclassification of mellow sickness as ordinary because of the CNNs failure to identify unobtrusive infection highlights. They foundthat preprocessing with differentiate constrained versatile histogram adjustment and guaranteeing dataset loyalty by master confirmation of class names improves acknowledgment of unaffected highlights. Authors M.Arora, M.Pandey,(2019) suggested that, The execution ofConvNet based algorithm for diabetic retinopathy characterization from fundusimages. Their work shows the practicality of deep learning way 
to deal with this problem. They conquer the issue by utilizing deep learning calculation which consequently recognizes the pattern and categorizes the retina images into five class based. Authors S.Kajan, J.Goga, KristianLacko, J.Pavlovi,(2020)is proposed a programmed detection of diabetic retinopathy and using a pretrained convolutional neural system (CNN). From Diabetic Retinopathy Database the retinal images, which was separated into five classes dependent on evaluation of retinal harm. images preprocessed and used as training and testing information from this database. At the order precision of three distinctive pre-trained neural network they compared and select the good one. AuthorsL. Jain, S. Murthy, C.Patel, D.Bansal (2018)proposed in this research that without executing clear segmentation or highlight extraction they will arrange images with retinal issues. For sound or sick retinal fundus images a deep learning model is used. Their objective of the venture was to build up a framework to arrange the retinal images as infected or solid. They have built up a framework known as LCDNet by using convolution neural systems. That effectively executed this binary classification. AuthorsY.Hui Li, Nai-NingYeh, K.Purwandari, (2019) proposed a PC supported output for programmed diabetic retinopathy analysis which is apply fragmentary max-pooling technique in both (Deep convolutional neural network ) DCNN organize furthermore, TLBO (Teaching-learning-based optimization) improved SVM on arrangement stage after system training. They were applying the novel characterization strategy dependent on the most recent progression in deep learning and PC vision on this issue. Authors Y. Luo, J.Pan, S.Fan , Z.Du , And G.Zhang (2020)suggested that SFCN which is skilled by three fundamental phases. In first phase a few deformed convolutional layers to remove $\mathrm{CNN}$ depiction from retinal image to accomplished the future learning module, and to remake the given retinal image by using numerous deconvolutional layers. This module guarantees the learned element depiction contains adequate data to develop itself. In second phase preparation management is provided by the, anticipated consequence of fuzzy bunching calculation. At long last, they plan how to apply the all-around prepared self-directed system on retinal picture grouping task.

$\mathrm{z}=\mathrm{f}(\mathrm{x})$, In feature learning module from retinal image shows they obtain feature vector

f using Li,e Encoder by stacked through their limitations of convolution layers.

$\mathrm{z}=[\mathrm{h}(\mathrm{L}) 1, \ldots \ldots, \mathrm{h}(\mathrm{L}) \mathrm{mL}]$

$\mathrm{ml}$ is a features map calculated. [10]

Lre $=1 / \mathrm{N} \quad \sum\|\mathrm{x}-\mathrm{x}\|^{2}$

ExeX

Lre is reconstruction Loss.In fuzzy self-supervision module,[10]

$$
\begin{gathered}
\text { Lss }=1 / \mathrm{N} \sum\|\mathrm{fss}(\mathrm{Z})-\mu\|^{2} \\
\operatorname{Ex} \varepsilon \mathrm{X}
\end{gathered}
$$

They achieved Lss self-supervision loss ,[10]

$$
\begin{aligned}
& \mathrm{NC} \\
& \sum \mu \mathrm{i}=1, \forall \mathrm{i}=1, \ldots \ldots \mathrm{Nc}
\end{aligned}
$$

$\mathrm{i}=1$, main components of retinal e of two types for single feature vector, as well as Nc clusters c D [c1; : : : ; ci; : : $: ; \mathrm{cNc}]$, and the chances be appropriate to per cluster u D [u1;:: : ui; : : : uNc].[10]

$$
\begin{gathered}
\mathrm{Lfs}=1 / \mathrm{N} \quad \sum \quad \sum \mu_{\mathrm{i}}^{\mathrm{m}}\left\|\mathrm{z}-\mathrm{C}_{\mathrm{i}}\right\|^{2}, 1 \leq \mathrm{m}<\infty \\
\text { Ex\&X } \mathrm{i}=1
\end{gathered}
$$

Lfs is Fuzzy supervision Loss.[10]

$\mathrm{L}=\mathrm{Lss}+\alpha \mathrm{Lss}+\beta \mathrm{Lfs}$

$\mathrm{L}$ is the loss function (final) of SFCN in total loss alfa, beta are balance parameter.[10]

To prepare SFCN system, they plan the two-phase streamlining calculation to direct: (1)In SFCN, to set up the underlying boundaries by train the encoder-decoder system. (2) In back creating way they updated the combined 
arrange by the fuzzy self-administered module. And unsupervised retinal image classification is focused bythese three sections as feature learning section, reconstruction module etc. Authors S Qummar, FG Khan, S Shah, A Khan (2019)proposed a system with data set, network architecture and training method. They pre-processed and augmented on fundus images obtained from public resources and used to train their model. By using deep learning method a new CNN model is developed which automatically detect RDR .To evaluate output of medical investigation and performance of binary classifier for that they used two parameters Sensitivity and Specificity. Sensitivity signifies the positive example as positive and Specificity signifies the negative examples as same. Explanation is as given below:

$\mathrm{TP}$

Sensitivity $=$ $\mathrm{TP}+\mathrm{FN}$
TN

(7)

where TP shows True positive examples that detected DR images correctly. FP shows false positive examples that detected No DR images, FN shows the false negative examples that detected number of images not correctly detected and TN shows true negative examples that detected correctly DR images.The Quadratic weighted Kappa is used for multiclass classification.[12]

$$
\mathrm{k}=1-\frac{\sum_{\mathrm{i}, \mathrm{j}} \mathrm{W}_{\mathrm{i}, \mathrm{j}} \mathrm{O}_{\mathrm{i}, \mathrm{j}}}{(\mathrm{i}-\mathrm{j})^{2}}, \mathrm{~W}_{\mathrm{i}, \mathrm{j}=}
$$

$$
\sum_{\mathrm{i}, \mathrm{j}} \mathrm{W}_{\mathrm{i}, \mathrm{j}} \mathrm{O}_{\mathrm{i}, \mathrm{j}} \quad(\mathrm{N}-1)^{2}
$$

Difference between fact and expected output is calculated by w is an 5x5 matrix of weights [11].Authors suggested that in color fundus images they sense and classify the DR's different stages through CNN. They train and test their model from kaggel dataset. Their output display that their model is used for detecting all stages of DRand as well as performs and compared to another model through proposed ensemble model.

\section{Case1:Deep Learning Approach}

A proposed T Chandrakumar, R Kathirvel (2016) Deep learning approach, for example, Deep Convolutional Neural Network(DCNN) gives exact through spatial examination. The supervised algorithm and preprocessing techniques are used for classification of images. Their proposed architecture carried with dropout layer procedures yields around 94-96 percent exactness. Additionally, they tried with mainstream databases, for example, STARE, DRIVE, kaggle fundus pictures datasets are openly to all. M.Hajabdollahi, (2019) By using proposed method that DR classification was obtained from hierarchical pruning a CNN structure. The suggested pruning method which was applied when VGG16-Net was improved with structure in FCLs. On different levels including tall filters, network connection and filters channels pruning method was applied. On Messidor image database gives appropriate accuracy than convolution filter in case of DR classification.

\section{Case2:Image Classification}

Authors T.Nazir , A.Javed , M.Masood ,J. Rashid,S.Kanwal (2019) proposed a technique that by using the, texture and color etc. Features classify several images for diabetic retinopathy. Their method gives better result. That includes various methods. The pre-processing shows the conduction of RGB is converted into $\mathrm{YCbCr}$ is presented in equation given below.

$$
\begin{aligned}
& Y=0.299 * r+0.587 * g+0.114 * b \\
& C b=0.866 * b-0.299 * r-0.587 g \\
& C r=0.701 * r-0.587 g-0.114 * b
\end{aligned}
$$

From the chrome portion $\mathrm{YCbCr}$ is used for decoupling of the segment. Feature Extraction include a set of feature i,e texture ,shape, color, texture and luminance. Proposed Gaussian model shown below for saliency $E_{l}^{n}$.

(L, Cb, Cr, T, D). m 1, Image spot is ' 1 ' with ' $\mathrm{n}$ ' 
T.Nazir , A.Javed , M.Masood et.al, (2019) features, whereas, $U_{l m}^{n}$ shows the distinction of highlight between patches.From above related work still there is scope of challenge and need modification

\section{Proposed Deep Learning Model for Image Classification}

From the Retinal image dataset several images are taken. The various features are taken into consideration from images. The next step is image pre-processing. To improve the image data Pre-processing is used which can defeat undesirable distortions or to improve image features which are essential for processing in advance. This step is essential to make the processing simpler. Next, the images are segmented to differentiate between into various classes. After pre-processing, the next step will be Feature Extraction, in which various features are extracted from image data eg.Optic Distance, Blood Vessel, Shannon Entropy Exudates Area,etc. and a quantitative analysis of these features is done.

The extracted features from the image will be selected and classified by using the Deep Learning algorithm. These features are classified into various classes. The resulting accuracy of each class and the overall accuracy of the class will be improved.

In this way, we will develop and improve the blindness detection technique by using python 3.5 and libraries are numpy, panda, matplotlib, Scikitlearn, Seaborn or any other tool as per requirement of research.

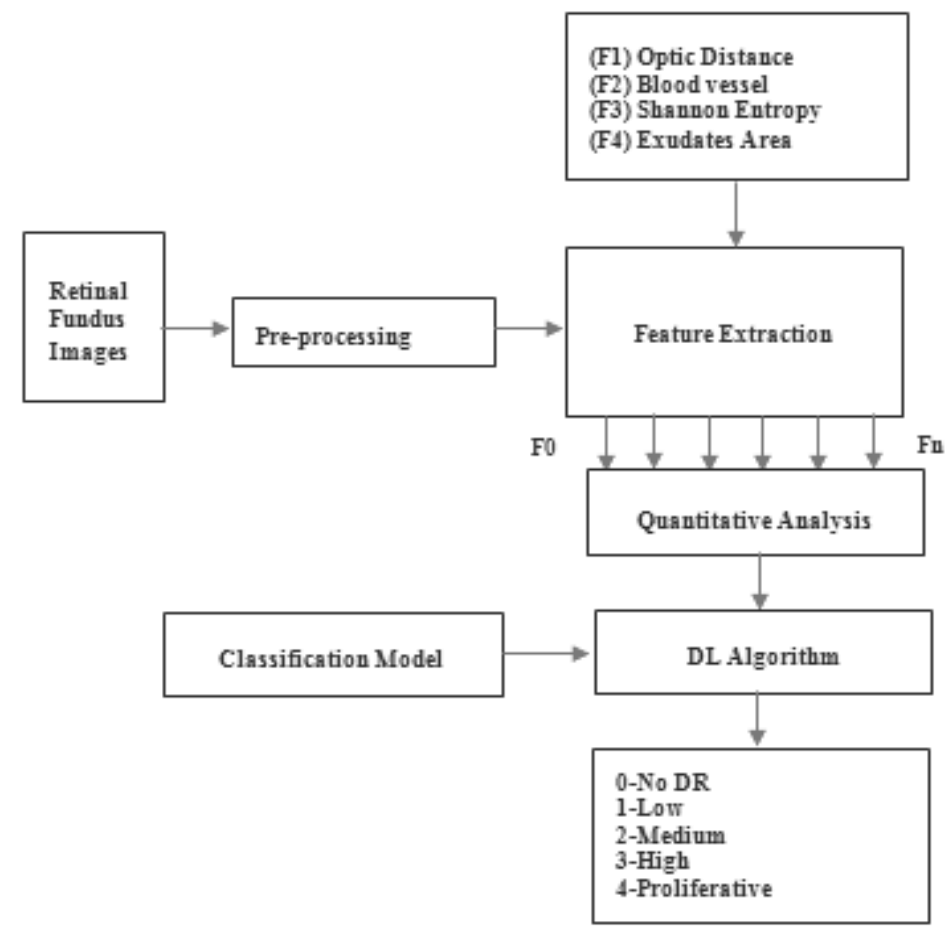

Figure 2. Proposed Deep Learning Model for Image classification

\subsection{Algorithm for Proposed model}

Step 1: capture various Retinal Images as a input.

Step 2: Then pre-processing from Retinal Image data.

(1) Pixel brightness transformations- brightness corrections and grey scale transformations.

(2) Geometric transformations of images.

- Such as Rotation, Scaling, Translation.

(3) Local neighborhood.

(4) Image Restoration

Step 3: Apply following Deep Learning model for classification

(1) CNN-Convolution neural network

(2) RNN-Recurrent neural network 
(3) RLNN-Reinforcement Learning to Neural Networks

Step 4: Performance Evaluation matrix

- $\quad$ Training and testing

Step 5:Result and Analysis

\subsection{Python code Snippet for the proposed algorithm}

a) Python code for reading an image in preprocessing

import pandas as pd

import numpy as np

import matplotlib.pyplot as plt

$\%$ matplotlib inline

from skimage.io importimread, imshow

image $=$ imread(image_image1.png', as_grey=True)

imshow(image)

b) reading an image file using matplotlib into numpy array

imp $=$ mpimg.imread ('../input/images/image1.jpg')

plt.imshow(img,dmap='retina image 1')

plt.scatter(dx,dy) \# show its center

plt.show ()

c) Feature Extraction code for LBP- Local binary patterns

From skimage import feature

import numpy as np

class LocalBinarypatterns:

def_init_(r,s, np):

self numpoints $=\mathrm{np}$

selfradius $=r$

def describe(s,image,eps=le-10):

$\mathrm{lpb}=$ feature Local_Binary_patterns(image,selfnumpoints,selfradius,method="uniform")

his=his as type("float")

his/=(his.sum ()$+$ eps

return his

d)python Code for classification

Logistic Regression 
From sklearn.linear_model import logisticRegression

lr=LogiticRegration()

lr.fit(x_train,y_train)

y_pred=lr.predict(x_test)

Random Forest

Form sklearn.ensemble import RandomForestClassifier

Rfc $=$ RandomForestClassifier ( $n \_$estimators $=20,11 b \_s c o r e=$ True, $n \_$jobs $=2$,

random_state $=109$, max_features $=$ none,min_samples_retina $=20$ )

rfc.fit(x_train, y_train)

y_pred=rfc.predict(x_test)

\section{Results and Discussion}

In our study some of the leading deep learning based algorithms are analyzed on various parameters such as algorithm, features, methods, database and result and summary of these are shown below in table 1

Table 1. Comparison of various deep learning-based algorithms for Image Classification, extraction and enhancement.

\begin{tabular}{|c|c|c|c|c|}
\hline Algorithm & Features & Methods & Database & $\begin{array}{l}\text { Result/Accurac } \\
\text { y }\end{array}$ \\
\hline $\begin{array}{l}\text { DL-based } \\
\text { segmentatio } \\
\text { n models [1] }\end{array}$ & \begin{tabular}{l}
\multicolumn{2}{l}{ Hemorrhages } \\
(HE), hard \\
exudates (EX) \\
and \\
Microaneurysms \\
(MA),etc. \\
\end{tabular} & $\begin{array}{l}\text { DR grading, } \\
\text { Dataset collection, } \\
\text { Annotation method, } \\
\text { etc. }\end{array}$ & $\begin{array}{l}\text { https://github. } \\
\text { com/nkicsl/D } \\
\text { DR-dataset } \\
\text { link. }\end{array}$ & $82.84 \%$ \\
\hline $\begin{array}{l}\text { CNN } \\
\text { Architec } \\
\text { ture [2] }\end{array}$ & $\begin{array}{l}\text { Very small } \\
\text { lesions }\end{array}$ & $\begin{array}{l}\text { CNN Architectures, } \\
\text { Preprocessing, } \\
\text { DataAugmentation,Tr } \\
\text { aining and Testing } \\
\text { Models, } \\
\text { Transfer Learning }\end{array}$ & $\begin{array}{l}\text { Messidor-1 } \\
\text { dataset } \\
\text { Kaggle } \\
\text { dataset }\end{array}$ & $95 \%$. \\
\hline $\begin{array}{l}\text { ConvNet } \\
\text { based } \\
\text { algorithm[3] }\end{array}$ & Hemorrhages & $\begin{array}{l}\text { Preprocessing and } \\
\text { Augmentation, } \\
\text { Convolutional Neural } \\
\text { Network- } \\
\text { Convolution Layer, } \\
\text { Pooling Layer, } \\
\text { Logistic Classifier. } \\
\end{array}$ & $\begin{array}{l}\text { ConvNet, } \\
\text { Kaggle }\end{array}$ & $74 \%$ \\
\hline $\begin{array}{l}\text { Pretrained } \\
\text { convolutiona } \\
\text { I neural } \\
\text { network }\end{array}$ & Some lesions & $\begin{array}{l}\text { Pretrainet Deep } \\
\text { Neural Networks, } \\
\text { Transfers Learning }\end{array}$ & $\begin{array}{l}\text { EyePacs } \\
\text { Diabetic } \\
\text { Retinopathy } \\
\text { Database }\end{array}$ & $93.37 \%$ \\
\hline
\end{tabular}




\begin{tabular}{|c|c|c|c|c|}
\hline$(\mathrm{CNN})[4]$ & & & & \\
\hline (LCDNet)[5] & $\begin{array}{l}\text { Optic disk, } \\
\text { retinal } \\
\text { vasculature, } \\
\text { macula and } \\
\text { fovea }\end{array}$ & $\begin{array}{l}\text { Dataset, } \\
\text { Image Cropper, } \\
\text { Image Resizer, } \\
\text { Image Augmentation, } \\
\text { Training using Deep } \\
\text { CNN, } \\
\text { Validation and } \\
\text { hyperparameter } \\
\text { setting, } \\
\text { Testing and } \\
\text { Prediction }\end{array}$ & $\begin{array}{l}\text { Friedrich- } \\
\text { Alexander } \\
\text { University } \\
\text { machine } \\
\text { learning data } \\
\text { repository, } \\
\text { Local eye } \\
\text { hospital } \\
\text { inBangalore, } \\
\text { India }\end{array}$ & $\begin{array}{lll}96.5 & \text { to } \\
99.7 \% . & & \end{array}$ \\
\hline Deep CNN[6] & $\begin{array}{lr}\text { vessels } & \text { and } \\
\text { symptoms } & \text { on } \\
\text { fundus edge } & \end{array}$ & $\begin{array}{l}\text { Preprocessing- Image } \\
\text { size normalization by } \\
\text { scaling, } \\
\text { Image boundary } \\
\text { removal. } \\
\text { Fractional Max- } \\
\text { Pooling, SVM }\end{array}$ & Kaggle & $74 \%$ \\
\hline $\begin{array}{l}\text { DL Based } \\
\text { algorithm }\end{array}$ & $\begin{array}{l}\text { Blood vessal part } \\
\text {,Excudates part } \\
\text { Microaneurysms } \\
\text { part }\end{array}$ & $\begin{array}{l}\text { Pre-processing } \\
\text {,feature extraction, } \\
\text { quantitative analysis }\end{array}$ & Kaggle & $98 \%$ \\
\hline
\end{tabular}

We have discussed about the current methodologies to identify diabetic retinopathy in the above section. Diabetes happens when the degree of glucose in the blood is get high over the typical. Over certain years diabetes can harm the veins of the retina which cause likewise called diabetic retinopathy (DR) and is the significant reason for helpless vision and visual deficiency. In early stages if the disease is diagnosed, treatment can prevent the development and which can be delayed. The programmed location of diabetic retinopathy helps the ophthalmologists for giving better treatment to their patients. Every one of the procedures talked about CNN because of its high accuracy, but having limited set of image features.

In our proposed algorithm we extract different features such as Microaneurysm, Macula ,Blood Vessel, optic disk taken from retinal images by preprocessing. Classify all features into various classes to detect the severity of Diabetic Retinopathy by improving accuracy of each class.

\section{Conclusion}

In this paper we have done a detailed analysison various Deep Learning Algorithms for diabetic retinopathy and as well as we have proposed an alorithm for early detection of DR. Diabetic retinopathy is a very danger in present situation. Early diagnosis of diabetic retinopathy is extremely basic and significant for avoidance of vision harm. Large part of population are affected by diabetic retinopathy (DR). Accordingly the above section included the current methods for the automatic valuation.During invetigations, it is found that DLbased segmentation models and detection modelsgives $82.84 \%$ accuracy by using haemorrhages (HE), hard exudates (EX) and microaneurysms (MA)these features gives marked.InCNN Architectureresults $95 \%$ using very small lesions ,pretrained convolutional neural network (CNN)[4] results $93.37 \%$ using some lesions and (LCDNet)results $96.5 \%$ to $99.7 \%$ accuracy depicts highest results among all by using optic disk, retinal vasculature, macula and fovea type features. In deep CNN results $74 \%$ accuracy by using vessels and symptoms on fundus edge and ConvNet based algorithmresults $74 \%$ using Hemorrhages deduced as compared to other shown algorithms.In conclusion there is no standard algorithm for detecting accuracy of each class. At prelim stage our proposedalgorithm is giving better results and accuracy in the range of $98 \%$ to $99 \%$ for 
enhancing a accuracy of each class by using Deep learning algorithm. By taking retinal image from database, then preprocessing of image data followed by Feature extraction and classification. At last algorithm will be trained on training data and tested. After implmentation on real data. This proposed algorithm become more reliable, accurate by introducing new feature as well as by providing better quality input by using advanced algorithm of image processing.

\section{References}

1. T. Li a,b , Y.Gaoa, K. Wanga,c, S.Guoa, H.Liud, H.Kanga,e, "Diagnostic assessment of deep learning

2. algorithms for diabetic retinopathy screening", volume 501,in Elsevier 2019.

3. C. Lam, MD, D.Yi, M.Guo, T.Lindsey, "Automated Detection of Diabetic Retinopathy using Deep

4. Learning", Articles from AMIA Summits on Translational Science Proceedings are provided here courtesy of American Medical Informatics Association Nov. 2018.

5. M.Arora, M.Pandey, "Deep Neural Network for Diabetic Retinopathy Detection", International

6. Conference on Machine Learning, Big Data, Cloud and Parallel Computing (Com-IT-Con), India,IEEE, Faridabad,India 14th - 16th Feb 2019.

7. S.Kajan, J.Goga, KristianLacko, J.Pavlovi "Detection of Diabetic Retinopathy Using Pretrained Deep

8. Neural Networks", IEEE, 19 Mar 2020.

9. L. Jain, S. Murthy, C.Patel, D.Bansal, "Retinal Eye Disease Detection Using Deep Learning", Bangalore,

10. India, IEEE 2018.

11. Y.Hui Li, Nai-NingYeh, K.Purwandari, "Clinically Applicable Deep Learning for Diagnosis of Diabetic

12. Retinopathy", Twelfth Intsernational Conference on Ubi-Media Computing (Ubi-Media), IEEE ,2019.

13. A.Guptaa, R.Chhikarab,"Diabetic Retinopathy: Present and Past", in International Conference on

14. Computational Intelligence and Data Science (ICCIDS) ScienceDirect, Procedia Computer Science 132 (2018)

15. M. K V, A. K S,"Detection of Retinal Lesions Based on Deep Learning for Diabetic Retinopathy",

16. IEEE,2019.

17. A.Shah, S.Lynch, M.Niemeijer, R.Amelon,W.Clarida ; J.Folk ,S.Russell ,X.Wu ; Michael,"Susceptibility

18. to Misdiagnosis of Adversarial Images by Deep LearningBased Retinal Image Analysis Algorithms", 15th International Symposium on Biomedical Imaging, Washington, D.C., USA , IEEE 2018.

19. Y. Luo, J.Pan, S.Fan , Z.Du, And G.Zhang ,"Retinal Image Classification by Self-Supervised Fuzzy

20. Clustering Network", IEEE access 2020.

21. X.Zeng, H.Chen, Y.Luo,And W.Ye,"Automated Diabetic Retinopathy Detection Based On Binocular

22. Siamese-Like Convolutional Neural Network", Special SectionOn Deep Learning For Computer-Aided Medical Diagnosis, IEEE ,1 February 2019.

23. S Qummar, FG Khan, S Shah, A Khan"A Deep Learning Ensemble approach by diabetic retinopathy

a. Detection", Volume: 7 ,IEEE, Year: 2019 .

24. T Chandrakumar, R Kathirvel,"Classifying Diabetic Retinopathy using Deep Learning Architecture",

25. International Journal of Engineering Research \& Technology (IJERT), Issue 06, Vol. 5, June-2016.

26. M.Hajabdollahi, "Hierarchical Pruning For Simplification Of Convolutional NeuralNetworks In Diabetic

27. Retinopathy Classification", 2019, IEEE.

28. T.Nazir, A.Javed, M.Masood ,J. Rashid,S.Kanwal, "Diabetic Retinopathy Detection based on Hybrid

29. Feature Extraction and SVM",13th International Conference on Mathematics, Actuarial Science, Computer Science and Statistics (MACS) 2019.

30. W AEzat, M MDessoukyand N A Ismail,"Multi-class Image Classification Using Deep learning CNN

31. model",Journal of Physics: Conference Series,ICaTAS 2019.

32. T.Soomro (Member, Ieee), Ahmed J. Afifi, L. Zheng, S.Soomro, J.Gao , O.Hellwich, And M. Paul "Deep

a. Learning Models for Retinal Blood Vessels Segmentation:: A Review", IEEE 2019.

33. D.Sisodia, S.Nair And P. Khobragade,"Diabetic Retinal Fundus Images: Preprocessing and Feature

34. Extraction For Early Detection of Diabetic Retinopathy",Biomedical \& Pharmacology JournalVol. 10(2), (2017).

35. M. Zile,"Creating Ergonomics Risk Analysis Algorithm and Risk Assessment Software Based Fuzzy

36. Logic In Hospitals"Issue 37,Vol10,No-4,pp65-69,Dec18.

37. M. Topal, I. Iskender, N. Genc"Sensorless Speed Control of a Bldc Motor Using Improved Sliding Mode

38. ObserverTechnique",Issue38,Vol11-No1,pp1-9,Mar2019. 